\title{
Wrinkling of a nanometric glassy skin/crust induced by drying in poly(vinyl alcohol) gels
}

\author{
Karine Huraux, Tetsuharu Narita,* Bruno Bresson, Christian Frétigny and François Lequeux
}

Received 29th February 2012, Accepted 30th May 2012

DOI: $10.1039 / \mathrm{c} 2 \mathrm{sm} 25480 \mathrm{~h}$

During drying of a chemically crosslinked poly(vinyl alcohol) gel, we observed, by atomic force microscopy (AFM) and scanning electronic microscopy, micron-order wrinkling which remains until the end of drying. We show that while at high humidity drying is homogeneous and no surface instability is observed, fast drying at low humidity induces the formation of a glassy skin layer on the soft bulk that wrinkles because the skin is compressed as the bulk contracts. The role of the glass transition is not only to create a mismatch of the elasticity with the bulk but also to remove the in-plane tensile tension which stretches the skin before drying. We proved experimentally the presence of this thin glassy skin on the wet bulk by approach-retract curve measurements in AFM. We also comment on the effect of mechanical restraint and the observation of hierarchical wrinkling over different length scales.

\section{Introduction}

Wrinkling of thin elastic matters has been observed for various systems in industry as well as in nature. ${ }^{1}$ Efforts have been made recently to understand the underlying mechanism of this phenomenon. It is interesting to use wrinkling in order to create controlled periodic surface morphologies that may be useful in many applications: among others we can quote microfluidic, cell culture or functional coatings. It is also important for certain systems to avoid wrinkling, such as in paintings, or our skin which is dehydrated or largely stretched, for instance.

Wrinkling is likely to occur on a system made of a thick and soft under-layer topped by a thin and stiff skin which is subjected to a compressive stress. This phenomenon has been widely observed for different systems and studied theoretically. ${ }^{2-7}$ It was studied for instance on poly(dimethyl siloxane) films exposed to ultraviolet/ozone radiation, plasma or a focused ion beam: ${ }^{8-11}$ the principle lies in the mismatch of coefficient of thermal expansion or of the strain between a stiff layer created by oxidation and its soft substrate. By stretching the film or controlling the relative motion of the substrate and the ion beam or the duration of exposition, the induced strain and, thus, the width, the amplitude and the length scale of the wrinkles can be controlled. Wrinkling is also observed for different bilayer systems such as metal capped polymers ${ }^{12-14}$ where the strain is induced by heating, twolayer polymeric coatings made of a glassy top layer and a rubbery bottom layer, ${ }^{15}$ cured coatings where the top stiff layer is due to a gradient of solidification in depth ${ }^{16,17}$ or ultrathin polymer films bonded to a compliant substrate. ${ }^{18-20}$

The hydrogels can absorb reversibly a large quantity of water and thus they can undergo a large volume change during swelling-deswelling. Transient pattern formation during swelling of hydrogels has been intensively studied, ${ }^{1}$ and several different motifs such as cusps, ${ }^{21,22}$ honeycomb like or hexagonal patterns,${ }^{23}$ have been reported. This transient pattern formation is governed by the kinetics of gel swelling: the surface swollen layer tries to expand, while it is fixed to the unswollen core, thus the mechanical mismatch compresses the swollen layer to wrinkle. The pattern disappears as the gel approaches to its equilibrium swelling. Different models based on elastic theory have been developed to describe the formation of these patterns. Tanaka and Fillmore were the first to study ${ }^{24}$ the kinetics of swelling, and their theory was then completed ${ }^{25,26}$ leading to the stress-diffusion coupling model. ${ }^{27,28}$ It was then shown that these transient patterns may become stable for constrained gels. ${ }^{29}$ Wrinkling/creasing instability of gel layers attached to a solid substrate during swelling has also been studied experimentally and theoretically, as a function of solvent quality and elastically active chain concentration. ${ }^{5,30,31}$

It is believed that deswelling or shrinking of hydrogels can induce mechanical instabilities. ${ }^{1}$ Volume phase transition of gels can induce similarly the mechanical instabilities during deswelling, leading to patterns looking like bubbles, bamboo or tubes. ${ }^{32}$ Mechanical instability induced by drying is a "reverse problem" of that induced by swelling. Cao et al. demonstrated theoretically that the deformation of a swelling/shrinking soft cylinder with a core-shell structure can trigger elastic instability. ${ }^{7}$ Still, as far as authors know, there are few reports on wrinkling of gels induced by drying although many previous studies which only focused on 
the drying of polymer solutions have been reported. ${ }^{33} \mathrm{We}$ can quote Rizzieri and co-workers ${ }^{34}$ who studied the wrinkling of drying gelatine films under large strain deformation leading to wrinkles along the stress direction. They attributed this phenomenon to a partial dehydration of the gelatin film surface, and adapted the theory of wrinkling to their system giving a simple relation between the wavelength of the wrinkles, the thickness of the skin and the ratio between the modulus of the soft and the stiff layers. They suggested that the formation of the stiff skin may be associated with a glass transition, however, it was not proved experimentally.

Here we report on wrinkling of poly(vinyl alcohol) gels (PVA gels) induced by drying. We show that the drying-induced morphology of the gel is due to the crossing of the glass transition while varying the solvent concentration. More precisely, we show that drying can induce wrinkling by forming a glassy skin layer which is compressed by the shrinkage of the core/bulk. Contrary to what is expected and described for solution drying, this glassy skin is no more under mechanical tension but is subjected to a compressive stress due to the drying of the soft core/bulk. The presence of a glassy skin is confirmed experimentally by approach-retract curve measurements in atomic force microscopy.

\section{Experimental section}

\section{Materials}

Polyvinyl alcohol (PVA, $M_{\mathrm{w}}=14000,100 \%$ hydrolysed), glutaraldehyde (GA, 25\% solution) and hydrochloric acid $(\mathrm{HCl}$, $35 \%$ ) used in this study were purchased from Janssen Chimica (Belgium), Sigma-Aldrich (France) and Fluka (France) respectively, and used as received.

\section{Gel synthesis}

PVA gels are prepared by crosslinking chemically PVA with GA under acidic conditions. A solution of PVA $(10 \mathrm{wt} \%)$ is prepared by dissolving PVA in hot water $\left(95^{\circ} \mathrm{C}\right)$ under stirring. The PVA solution and GA are mixed together with water in a test tube, then dilute $\mathrm{HCl}$ is added to the mixture to start the crosslinking reaction. The final concentration of PVA and the crosslinking ratio are adjusted to $8 \%$ and $5 \mathrm{~mol} \%$ (crosslinking ratio is given by the molar ratio between PVA hydroxyl functions and glutaraldehyde in feed), at $\mathrm{HCl}$ concentration of $0.07 \%$.

PVA gels are prepared in two geometries: cylinder and sheet. In order to obtain gel cylinders, hematocrit capillary tubes (75 mm in length, 1.1-1.2 $\mathrm{mm}$ in diameter) are used as mould. After gelation, gels are removed from the mould and kept in water. The diameter of the gels at equilibrium swelling is about $0.8 \mathrm{~mm}$, with the equilibrium PVA concentration of about $15 \%$.

In order to obtain gel films, two glass slides for microscopy are used as mould. The surface of the glass slides is cleaned using plasma, then treated with hexamethyldisilazane to reduce the adhesion of PVA gels. The PVA-water-GA- $\mathrm{HCl}$ mixture is spread between a treated glass and a cleaned glass separated by a spacer of $100 \mu \mathrm{m}$ thickness. When the gel is formed, the treated slide is removed easily and the wet film adhered on the cleaned glass is kept in water.
The obtained PVA gels were characterized at dried and swollen states. The dynamic mechanical analysis was used to measure the gel modulus during drying, from the equilibrium swelling state to the dry state. It is conducted using a DMA (TA Instruments) working in film tension clamp at a frequency of $1 \mathrm{~Hz}$ and an amplitude of $0.01 \%$. The temperature was set to a value of $T=30{ }^{\circ} \mathrm{C}$.

In order to determine the glass transition temperature, $T_{\mathrm{g}}$, of the dry PVA gels, the calorimetric measurements were carried out using a differential scanning calorimeter (DSC2920, TA Instruments). DSC traces were recorded between 20 and $130{ }^{\circ} \mathrm{C}$ with a heating rate of $5{ }^{\circ} \mathrm{C} \mathrm{min}^{-1}$ and a modulation in temperature of $0.5{ }^{\circ} \mathrm{C} \mathrm{min}{ }^{-1}$. The values of $T_{\mathrm{g}}$ were taken as midpoint temperatures. $T_{\mathrm{g}}$ of the dry PVA gels was measured to be $91^{\circ} \mathrm{C}$.

\section{Drying of gels}

Drying of PVA gel cylinders is performed under controlled humidity and ventilation. The gels are hung, or the upper end of the cylinder is glued on a support, the lower end is left free (Fig. 1a). The gels are dried in a drying chamber under a flow of $1.5 \mathrm{~m} \mathrm{~s}^{-1}$ measured at the surface of the gel and controlled humidity (16-90\%). Temperature and humidity are monitored through sensors. Weight change of PVA gels during drying is monitored by weighing on a balance (BP221S, Sartorius).

In order to study the effect of mechanical constraint on the morphology, another series of experiments is performed with the gels fixed to the constant length, or both ends of the gels were fixed on the support to keep the initial length, $L_{0}$, prior to drying (Fig. 1b). The weight change is monitored in the same manner with the hung gels. Change in the diameter and in the surface morphology of the drying gels is followed by a CCD camera equipped with a microscope objective. Traction force induced by drying is also measured for gels at the constant length: the upper end is connected to the balance which served as a strain gauge and the lower end of the gels is fixed to a support to keep the fixed length.

\section{Morphology of dried PVA gels}

The surface morphology of gels is probed by atomic force microscopy. We used a Nanoscope III microscope Dimension 3100 operated in tapping mode. The samples are scanned at a rate from 0.3 to $0.5 \mathrm{~Hz}$ using a cantilever tip with a constant (a) Free end

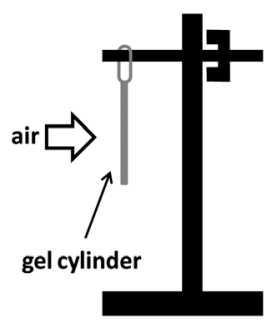

(b) Constant length

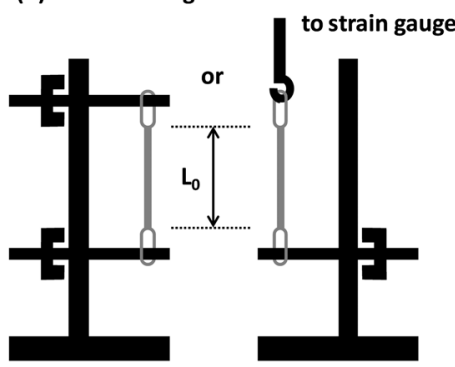

Fig. 1 Schematic illustrations of gel cylinder drying: (a) free end drying. A gel is glued and suspended from the support in a drying chamber. (b) Constant length drying. The gel length is fixed to its initial length $L_{0}$. Traction force is measured with a strain gauge. 
force of $40 \mathrm{~N} \mathrm{~m}^{-1}$ and a resonance frequency of about $300 \mathrm{kHz}$. They are also observed under a Hitachi S-3600 scanning electron microscope (SEM) operated with a beam energy of $5 \mathrm{keV}$.

We also used the Nanoscope III microscope Dimension 3100 to obtain the force-distance curves. The microscope is operated in contact mode at a scanning rate of $1 \mathrm{~Hz}$ using a cantilever tip with a force constant of $40 \mathrm{~N} \mathrm{~m}^{-1}$.

\section{Results and discussion}

\section{Drying-induced wrinkling}

In order to study the effect of humidity on the morphology of dried gels, a series of drying experiments of hung PVA gels with one end free in a cell under different humidity $h$ varying from 10 to $90 \%$ was performed. The final morphology of gels dried at $30 \pm 2{ }^{\circ} \mathrm{C}$ is observed under SEM and shown in Fig. 2. At high humidity $h=90 \%$ (Fig. 2a), no particular surface morphology was observed. At $h=66 \%$ (Fig. 2b), small bubble-like patterns appear on certain regions. At lower humidity ( $h=47 \%$, Fig. $2 \mathrm{c}$ and $h=16 \%$, Fig. 2d), we observed patterns due to wrinkling of the surface all over the sample. The wavelength of the wrinkling is about $0.5 \mu \mathrm{m}$ for $h=16 \%$ and $1 \mu \mathrm{m}$ for $h=47 \%$. The wrinkling has no specific orientation. The observed wrinkling is retained at the dried state, different from the pattern observed during swelling. Swelling the dried gel at $95{ }^{\circ} \mathrm{C}$ erases the wrinkling. At this temperature, dried gels recover their initial swelling degree, while at temperatures less than $95^{\circ} \mathrm{C}$, the swelling of gels is about $50 \%$ of the initial swelling. We believe that this higher swelling is due to both the melt state of the gel $\left(T_{\mathrm{g}}=91{ }^{\circ} \mathrm{C}\right.$ for the dry PVA gel) and the breaking of hydrogen bonds. ${ }^{35}$

We found a similar tendency for gels dried at constant length. Atomic force microscopy images are shown in Fig. 3. Wrinkles appear for low humidity and their wavelength decreases with the decrease in humidity. Here we see two generations of wrinkling: for $h=12 \%$ (Fig. 3c), we found the first generation with a wavelength of $1.5 \mu \mathrm{m}$, and the second with about $5 \mu \mathrm{m}$. We also observed that the wrinkles are orientated along the direction of the applied strain, indicating that the observed morphology is not cracking which is supposed to appear perpendicular to the strain direction.
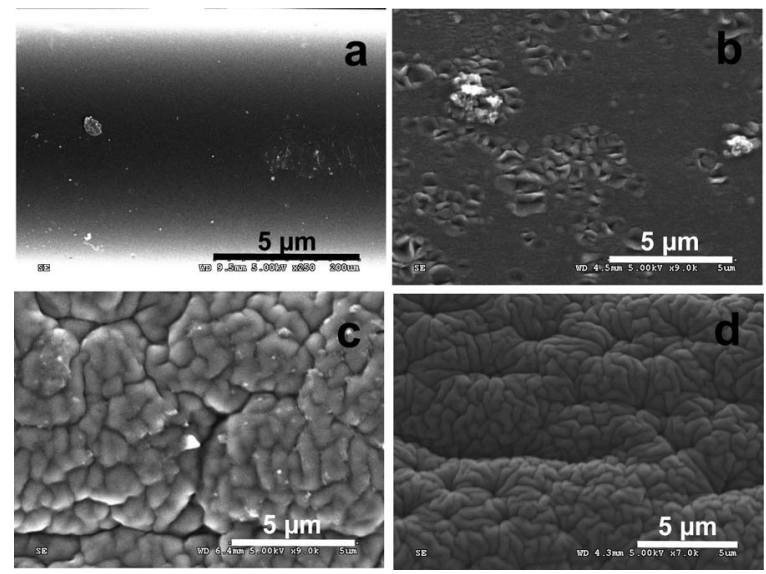

Fig. 2 Surface morphology of PVA gels dried with a free extremity under different values of humidity ((a) $95 \%$, (b) $66 \%$, (c) $47 \%$ and (d) $16 \%$ ) observed by SEM.
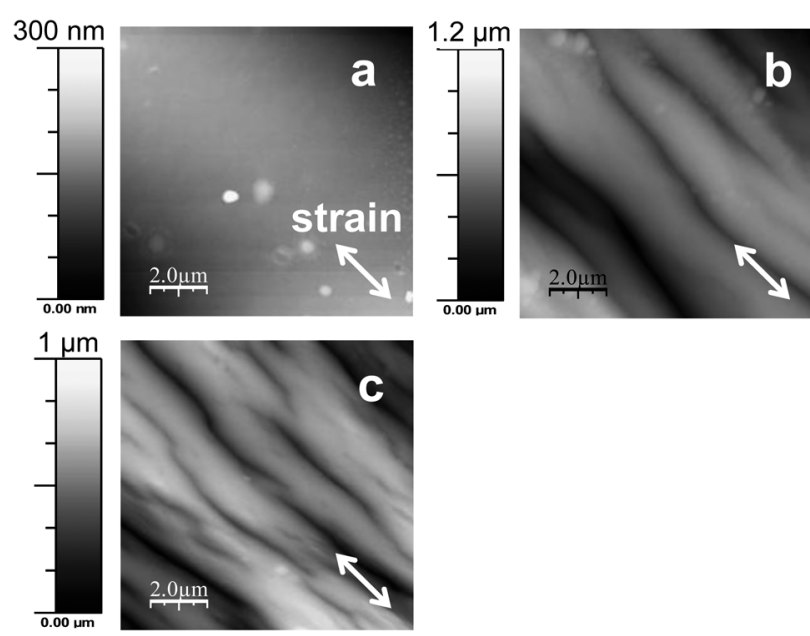

Fig. 3 Surface morphology of PVA gels dried at a fixed length under different humidities ((a) $85 \%$, (b) $45 \%$, (c) $12 \%$ ) measured by AFM.

According to the theory, ${ }^{2}$ wrinkling occurs (1) when there is an upper stiff layer on a soft support and (2) when this stiff layer is compressed in the in-plane direction (when the strain reaches a threshold depending on the thickness and the modulus of the upper layer and the modulus of the under layer, wrinkling occurs rather than compression). It is reasonable to consider that drying can induce a concentration gradient of the polymer and creates a concentrated polymer layer on the drying surface when the drying is fast enough as compared with equilibration by diffusion of water-polymer.

How does drying apply a compressive stress to the outer layer? Cao et al. predicted that for a soft cylinder with a core-shell structure, compressive stress can appear when the shrinkage of the core is larger than the shell. ${ }^{7}$ In the case of the drying gel cylinder, the surface layer (shell) always shrinks more than the bulk (core). In other words, the outer layer of the gel is submitted to a tensile stress: while the outer layer is drier and contracts, the bulk is not yet dried, the outer layer is stretched in the in-plane direction (compressed in the thickness direction). From the mechanical point of view, this is a reverse problem of the swelling gel: for swelling the surface layer swells more than the bulk thus the surface layer is compressed, while for drying the surface layer dries more than the bulk thus the surface layer is stretched. For either case the core is considered as incompressible.

In the drying gel cylinder, in order to compress the outer skin layer in the in-plane direction and in order to make it wrinkle, this tension has to be removed without any contraction of the skin. The only possibility is that the skin undergoes the glass transition. As soon as the skin undergoes a glassy transition, it becomes very rigid but with an equilibrium strain which corresponds to its strain just before the glass transition. Or, the reference state of the deformation is reset (the tensile stress is stocked in the glass). Then further drying in the bulk decreases its volume and results in compression in the in-plane direction, thus inducing wrinkling of the glassy skin. Thus the drying induces successively: (i) the concentration gradient of the polymer: since the surface dries faster than the bulk, and the skin cannot be deformed isotropically (as the bulk is not dried) thus stretched, (ii) the glass transition: the tensile stress to the skin is removed 
and (iii) the compression of the stiff layer occurs inducing the wrinkling: when the bulk starts to dry. This mechanism is schematized in Fig. 4.

In the theoretical work of Cao et al. core-shell structure without any tension before shrinking is considered. On the other hand, in our drying gel which is homogenous before drying (and after complete drying), partial drying is necessary to create a core-shell (skin-bulk) structure which results in generation of tension of the skin layer. Therefore glass transition which is not considered in the work of Cao et al. is required for our case.

Contrary to the prediction of De Gennes, ${ }^{36}$ no cracking is observed on the surface of gels. Indeed the formation of cracks on glassy polymer films formed by evaporation is due to the mechanical tension applied to the crust as its volume decreases whereas its horizontal dimensions have to remain the same. In our system, as the skin becomes glassy, its length is fixed and as the inner volume contracts, the skin is no more under mechanical tension. Numerous studies focused on the drying of polymer solutions. ${ }^{33,36}$ However they deal with liquids in different geometries that are sessile drops and spin coated films and the hypothesis of glassy skin is not experimentally proved.

In the rest of this paper, we discuss the following three points concerning the proposed wrinkling mechanism of the PVA gel: (1) drying-induced glass transition, (2) formation of a glassy skin on the soft bulk, and (3) compression of the glassy skin and apparition of the wrinkles.

(1) Drying-induced glass transition. In general the presence of a solvent decreases the glass transition temperature $T_{\mathrm{g}}$ of a polymer. Thus at a given temperature lower than $T_{\mathrm{g}}$ of the dry polymer, there is a concentration of solvent at which the glass transition occurs. Gravimetric measurements allow us to determine the solvent weight fraction as a function of the activity of the solvent which is equal to relative humidity in the case of water. Experimental details are given in ref. 37. Sorption/ desorption experiments can be performed by changing the vapour pressure of the solvent and by measuring the mass of the solvent absorbed/desorbed by a polymer film. Above the glass transition temperature of the polymer, the solvent weight fraction as a function of the activity of the solvent can be fitted by the Flory-Rehner theory. ${ }^{38}$ Below the glass transition temperature, on the contrary, at the low weight fraction of the solvent, the

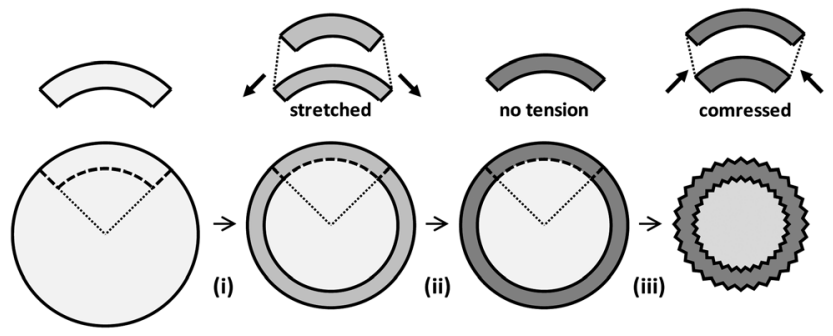

Fig. 4 Schematic representation of the drying mechanism. The crosssection of the cylinder is illustrated as a circle: (i) water evaporation inducing a concentration gradient and the formation of a skin under stretching, (ii) glass transition occurs and the tensile stress disappears, and (iii) shrinking of the bulk and compression of the stiff layer inducing wrinkling. glass transition occurs and the elasticity of the polymer increases, thus the desorption costs extra elastic energy and the deviation of the experimental curve from the Flory-Rehner fit can be observed. ${ }^{39}$ This excess sorption can be used to determine the glass transition weight fraction and solvent activity at the given temperature. At $30^{\circ} \mathrm{C}$, the solvent weight fraction corresponding to the glass transition was found to be $0.125 \pm 0.025$, at the humidity of $60 \pm 5 \%$. This value is reasonable compared with the estimation by the theories which calculate thermodynamically $T_{\mathrm{g}}$ as a function of the solvent concentration..$^{40,41}$

This result agrees well with the wrinkling shown in Fig. 2. We see that for relative humidity under $60 \%$, wrinkles are observed: the gel surface in equilibrium with the surrounding atmosphere may become glassy and wrinkle. Above $60 \%$, the surface of the samples may be considered as smooth. For the drying with a humidity of $66 \%$, which is very close to the activity necessary to induce the glass transition, the observed heterogeneous small patterns may be attributed to heterogeneities in crosslinking (Fig. 2b). DSC measurements show that $T_{\mathrm{g}}$ increases with the crosslinking ratio $^{42}$ so that we may assume that the solvent content inducing glass transition also varies with the crosslinking ratio. ${ }^{31}$ Thus parts of the gel are likely to become glassy and form some scattered patterns.

(2) Glassy skin on the soft bulk. How thin is this glassy skin layer? Considering the bending and stretching energies and geometrical constraints, Cerda and Mahadevan ${ }^{3}$ developed a general theory of wrinkling giving a simple relation between the wavelength of the wrinkles $\lambda$, the thickness of the skin $e$ and the ratio between the modulus of the soft and the stiff layers, respectively, $E_{\mathrm{m}}$ and $E_{\mathrm{s}}$ :

$$
\lambda \approx e\left(\frac{E_{\mathrm{S}}}{E_{\mathrm{m}}}\right)^{1 / 3}
$$

Because it is very difficult to measure the modulus of the drying surface and that of the bulk separately, we used the value of modulus of the completely dry gel for the drying surface, and that of swollen gel for the bulk. From the experimental values of the moduli $\left(E_{\mathrm{s}}=2.1 \mathrm{GPa}, E_{\mathrm{m}}=0.2 \mathrm{MPa}\right)$ and the wavelength of the wrinkles $(\lambda \sim 1 \mu \mathrm{m})$ the thickness of the skin is estimated to be $e \sim 50 \mathrm{~nm}$ which is very thin. In order to prove the presence of such a thin dry skin, an experiment at a nanometric scale is required. We performed AFM measurements as suggested by De Gennes. ${ }^{36}$

Approach-retract curve measurements in atomic force microscopy were performed to study mechanical properties of the surface of drying gels. These curves give the deflexion of the cantilever as a function of its distance to the gel surface. The AFM is operated in contact mode with a rather stiff cantilever: its force constant is high enough $\left(40 \mathrm{~N} \mathrm{~m}^{-1}\right)$ to be sensitive to the surface stiffness; let us remind that in this situation, adhesion cannot be measured. These approach-retract curves measured with a stiff cantilever, as well as experiments performed in tapping mode, ${ }^{43}$ show the difference between hard and soft samples: on a harder surface, the increase in the deflexion is more important thus the slope of the curve is larger than that on a softer surface. Although the technique is not quantitative, especially due to the concentration gradient in the drying gel 
surface, we can qualitatively discuss the evolution of the stiffness of the surface. As both the cylindrical geometry and the thickness of our gels are not convenient for these experiments, the samples used here are gel films with a thickness of about $60 \mu \mathrm{m}$ at equilibrium swelling.

We made two experiments with different drying conditions. The first experiment was performed at room conditions ( $h$ is about $60 \%$ ), in order to monitor the evolution of the viscoelasticity of a drying PVA gel film. In this condition, no wrinkling is observed either on a cylindrical gel or on a film. The swollen film is put on the AFM stage and approach-retract curves are measured during drying. Measured deflexion as a function of the distance from the gel surface is plotted in Fig. 5. The same experiment performed on a silicon wafer is shown as reference. The deflexion increases linearly with the distance from the surface once the tip is in contact with the gel and the value of the slope is an indication of the stiffness of the surface of the sample. After drying for more than 3 minutes, we observe the progressive increase in the slope of the approach curve, indicating that the gel surfaces are hardening. After 8 minutes, the slope is approaching to that of the dried glassy gel, suggesting that the surface is now glassy.

The second experiment is performed under airflow $\left(1.5 \mathrm{~m} \mathrm{~s}^{-1}\right.$, $h=16 \%$ ) in order to study the effect of the drying rate on the surface elasticity. The aim of this experiment is to show the presence of a glassy skin or its rewetting by performing alternatively fast and slow drying to favour respectively evaporation or homogenisation by diffusion in the gel. The curves are reported in Fig. 6. The dashed black and grey curves correspond respectively to the silicon and to a reference elastomer. The dotted black curve corresponds to the dried gel. The swollen gel is put on the AFM stage and is subjected to the dry airflow: the surface is instantly seen as stiff as a dry film (curve 1). After two minutes of drying, the airflow is stopped and the drying rate is lower. After two minutes without airflow, we observed a decrease in the slope (curve 2), indicating that the bulk rewets the surface glassy layer. As this cycle is immediately repeated, the same results are obtained: by applying the airflow the film is seen as a dried film (curve similar to curve 1), then it is rewetted two

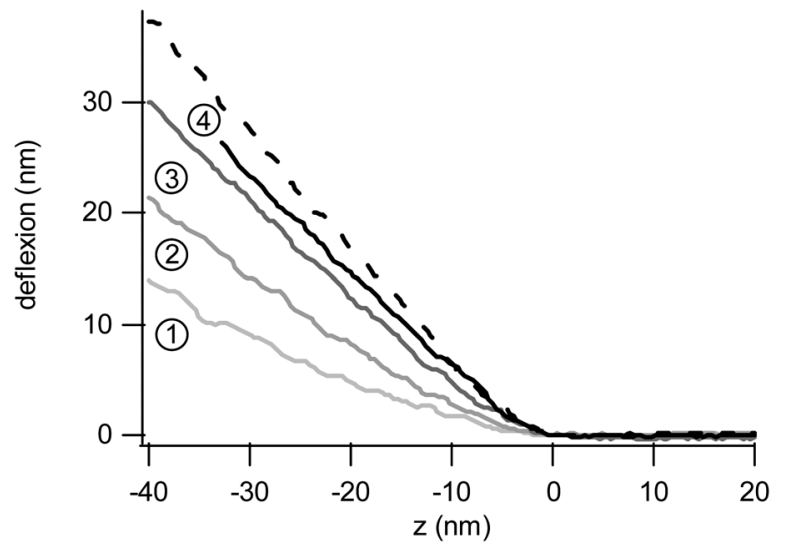

Fig. 5 AFM approach-retract curves of PVA gels under drying at room conditions. Curve 1: 3 min of drying, curve 2: 5 min of drying, curve 3: 8 minutes of drying, curve 4: dried gel. Dotted black curve corresponds to silicon used as reference.

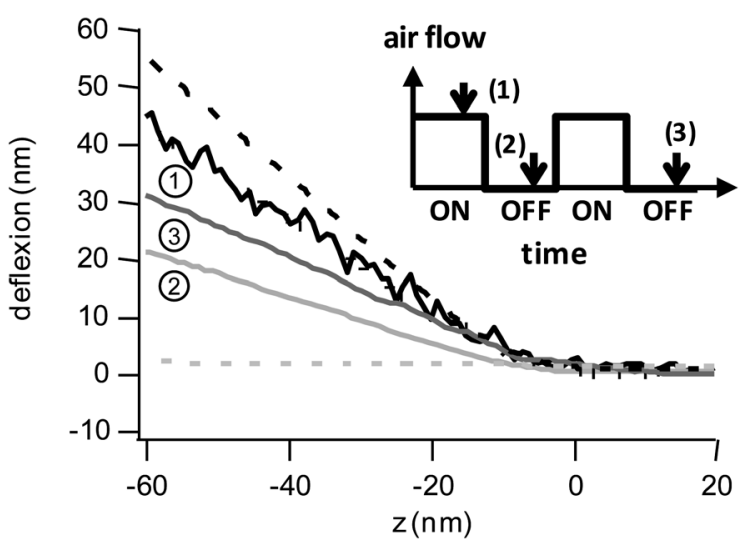

Fig. 6 AFM approach-retract curves of PVA gels under drying under airflow. Curve 1: under airflow, curve 2: 2 min after stopping airflow, curve 3: 2 min after stopping airflow. Dashed black and grey curves correspond to silicon and elastomer used as references, the dotted black curve corresponds to the dried gel.

minutes after stopping the airflow (curve 3). This result indicates that a dry glassy skin is formed on the bulk, less dried, thus soft, as required for wrinkling.

(3) Compression of the glassy skin. The mechanism of wrinkling for drying PVA gels is based on glass transition of the drying surface layer. The surface layer is under tension since the surface layer dries faster than the core/bulk in the beginning of drying (no wrinkling); then, after the glass transition, it is under compression (thus wrinkling occurs) since the glass transition resets the in-plane stretching tension, and the bulk starts to dry. In order to evaluate the effect of compression due to the volume change, for a drying PVA gel cylinder in the constant length (the natural length), the traction force to keep the length constant was monitored simultaneously with the surface morphology as well as the evaporation rate (estimated from the radius). This experiment allows us to correlate the formation of wrinkles with the evolution of the evaporation rate and the traction force, which are strongly influenced by the glass transition. Especially the traction force is sensitive to not only the volume change of the drying gel, but also eventual stress relaxation due to glass transition. A curve for a drying experiment at $h=57 \%$ is plotted in Fig. 7a. The force increases with time as the volume of the gel decreases and the equilibrium is obtained after more than 60 hours, probably due to the slow diffusion and evaporation of the residual water in the glassy PVA gels.

More importantly, we found that the increase of the traction force changes when the wrinkling occurs in the beginning of drying, coinciding with the decrease in the drying rate. The traction force curve, evaporation rate (calculated from weight loss and diameter change) and corresponding light microscopy images of the surface morphology in the beginning of drying are shown in Fig. 7b. We observed simultaneously a decrease in the slope of the force curve, a sharp drop in the evaporation rate, and an apparition of the wrinkles at about $15 \mathrm{~min}$ (Fig. $7 \mathrm{~b}$ and c). We attribute the slowdown of the stress increase at about $15 \mathrm{~min}$ to the glass transition of the gel surface and the relaxation of the force. The concomitant drop in evaporation can be explained by the low coefficient of diffusion of water in a glassy polymer. This 

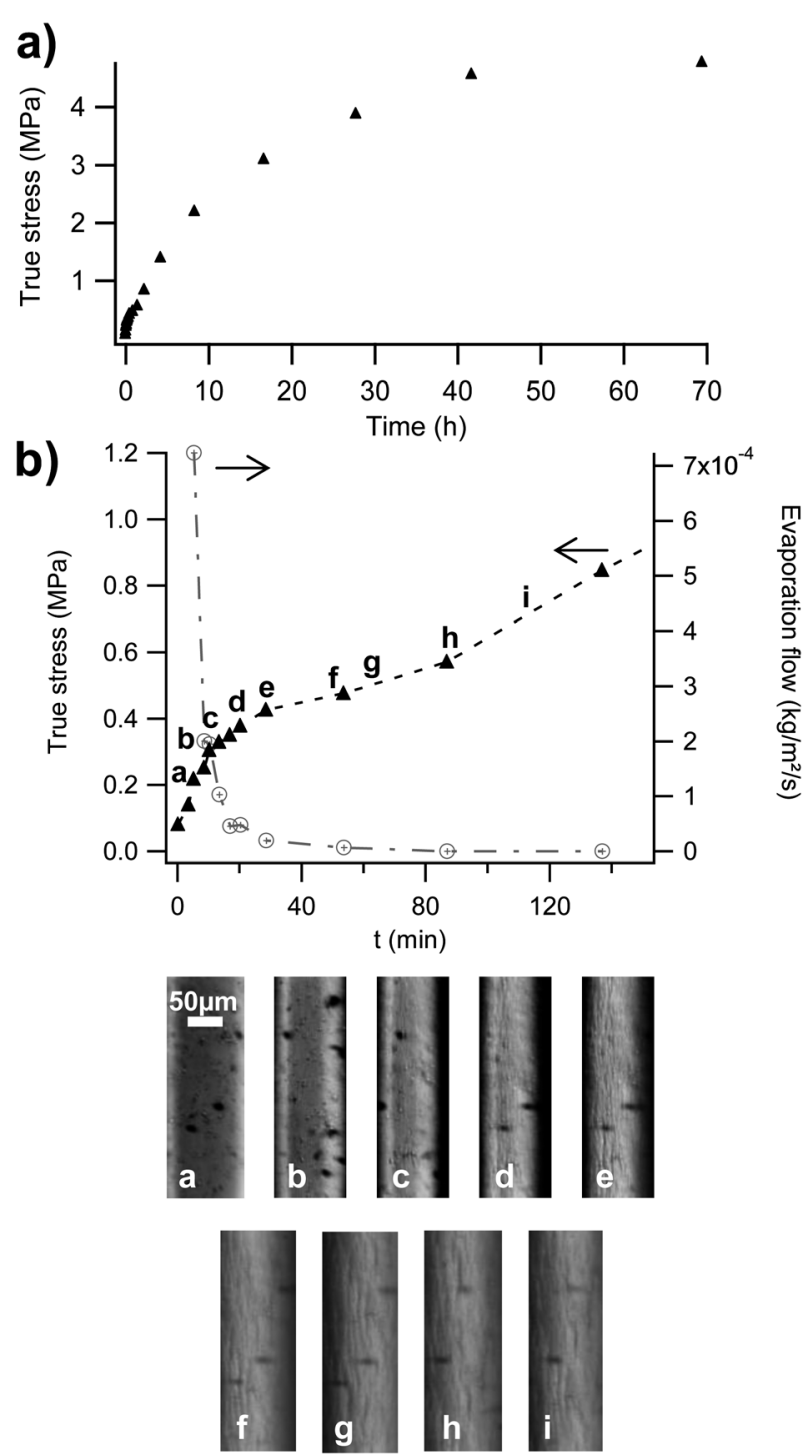

Fig. 7 (a) Time profile of the contraction force for drying $h=57 \%$; (b) time profiles of the contraction force (triangles), and the evaporation flow (circles) and imaging of gel surface by optical microscopy during drying at $h=57 \%$.

is also at the moment that the first wrinkles appear on the surface. Then, the traction force increases very slowly and the amplitude and wavelength of the wrinkles increase until the stress begins raising faster (Fig. 7b-f), suggesting that either the skin thickness $e$ or the elastic modulus $E_{\mathrm{s}}$ of the skin increases. Finally, the morphology is fixed and no more change in diameter is observed (Fig. 7b-i); we attend the evaporation of the residual water (Fig. 7a) and the rise of the modulus.

Let us comment on the following two points about the gels dried at constant length. Here we saw (1) that wrinkles are orientated along the direction of the applied strain and that for $h=12 \%$, and (2) that two generations of wrinkling occur: we found the first generation with a wavelength of $1.5 \mu \mathrm{m}$, and the second with about $5 \mu \mathrm{m}$.

The isotropic wrinkling when the lower extremity is free may be explained by the minimization of the Flory elastic energy ${ }^{44}$ given by

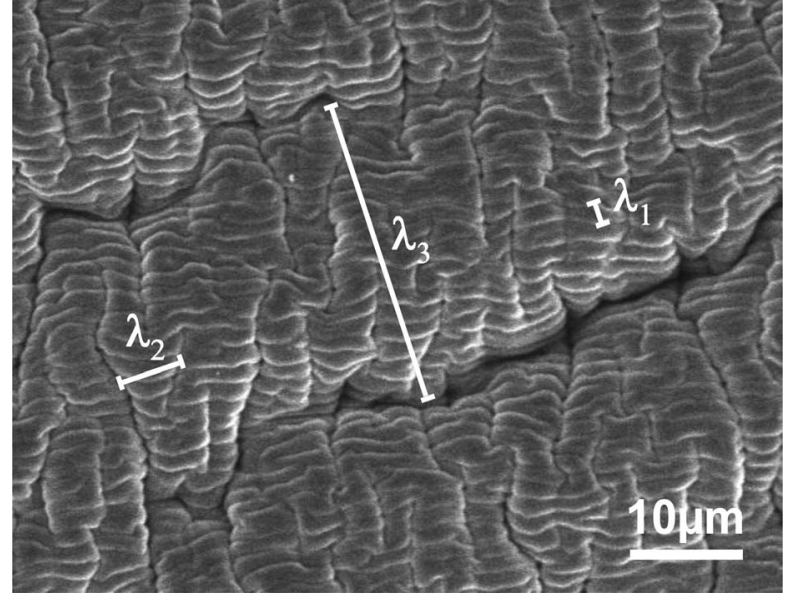

Fig. 8 Surface morphology of a PVA plate dried under an airflow.

$$
F_{\text {ela }}=k_{\mathrm{B}} T \sum_{i=1}^{v_{e}} \frac{1}{2}\left[\alpha_{i 1}^{2}+\alpha_{i 2}^{2}-2-\ln \left(\alpha_{i 1}^{2} \cdot \alpha_{i 2}^{2}\right)\right]
$$

which is minimum when the elongation ratios along the two principal axes of deformation $\alpha_{1}$ and $\alpha_{2}$ are equal. In contrast, if the lower extremity is clamped, the tensile strain applied along the length of the gel prevents it from buckling in the transverse direction so that a preferential orientation is observed along the strain direction.

Regarding the hierarchical wrinkling, it may be explained in the same manner as for drying gelatin gels or PDMS films: ${ }^{5,25}$ as drying continues, the skin thickens and hardens as it dries. Provided that the strain threshold is exceeded, this new skin buckles on a larger length scale as described by eqn (1). We also observed this hierarchical patterning on circular PVA plates (diameter $15 \mathrm{~mm}$, thickness $1 \mathrm{~mm}$ ) dried on a glass substrate under an airflow under $h=16 \%$ (Fig. 8). We found three generations of wrinkling having wavelengths of $1.2,5$ and $20 \mu \mathrm{m}$. Under the airflow, due to the faster drying of its upper face, the plate buckles macroscopically looking like a potato chip. Wrinkling of the smaller wavelength occurs perpendicularly to the direction of the macroscopic buckling and then, successive wrinkling appears perpendicularly to the previous one. The increasing wavelength of wrinkling is attributed to the thickening and hardening of the skin and their relative orientation corresponds to the successive relaxation. Once the plate has relaxed macroscopically, the skin is at equilibrium along the direction of bending whereas along the direction perpendicular to bending, it is still under compressive stress. To accommodate it, wrinkling occurs and its wavelength is larger as the skin thickens and hardens. This is repeated giving successive generations of wrinkling perpendicularly to the previous one.

\section{Conclusions}

In summary, we observed a phenomenon of wrinkling appearing on the surface of PVA gels during drying. Fast drying (inducing a concentration gradient) at low humidity (inducing glass transition) is required for the gels to wrinkle. The value of the wavelength of the wrinkles is about $1 \mu \mathrm{m}$ and increases with 
increase in the humidity. Stretching the gels orients the wrinkles. Since the wrinkles remain after drying, this morphology is the consequence of the formation of a glassy skin at the surface of the gel which undergoes a compressive stress induced by the drying of the bulk.

Some evidence for the presence of a glassy skin essential to the formation of wrinkles has been produced: AFM experiments have shown that if the drying is fast enough, the gel is composed of a dry upper layer on a wet bulk and traction force measurements have revealed that wrinkles appear when the glass transition occurs.

We qualitatively described the phenomenon of wrinkling on PVA gels. However, further studies focusing on the characterization of the skin formed as a consequence of heterogeneous drying in the direction of the diameter are required in order to better understand the phenomenon quantitatively. Experimentally a quantitative analysis becomes complicated because of the parameter interdependence: a change in crosslinking ratio modifies the glass transition temperature, the mechanical properties or the structure of the network (heterogeneities). That is why the thickness of the skin and its deformation during drying under different conditions should be theoretically analysed.

\section{Acknowledgements}

This work was partly funded by the grant DEPSEC of the Agence Nationale de la Recherche. The authors also thank B. Guerrier and F. Doumenc from FAST laboratory who performed and exploited the gravimetric experiments and F. Martin from PPMD laboratory for SEM imaging.

\section{Notes and references}

1 B. Li, Y.-P. Cao, X.-Q. Feng and H. Gao, Soft Matter, 2012, 8, 1301.

2 J. Groenewold, Phys. A, 2001, 298, 32-45.

3 E. Cerda and L. Mahadevan, Phys. Rev. Lett., 2003, 90, 074302.

4 J. Genzer and J. Groenewold, Soft Matter, 2006, 2, 310.

5 M. K. Kang and R. Huang, J. Mech. Phys. Solids, 2010, 58, 1582.

6 J. Dervaux and M. Ben Amar, J. Mech. Phys. Solids, 2011, 59, 538.

7 Y.-P. Cao, B. Li and X.-Q. Feng, Soft Matter, 2012, 8, 556.

8 N. Bowden, W. T. S. Huck, K. E. Paul and G. M. Whitesides, Appl. Phys. Lett., 1999, 75, 2557.

9 K. Efimenko, M. Rackaitis, E. Manias, A. Vaziri, L. Mahadevan and J. Genzer, Nat. Mater., 2005, 4, 293.
10 D. Breid and A. J. Crosby, Soft Matter, 2011, 7, 4490.

11 M. Watanabe, Soft Matter, 2012, 8, 1563.

12 N. Bowden, S. Brittain, A. G. Evans, G. Hutchinson and G. M. Whitesides, Nature, 1998, 393, 146.

13 T. Okayasu, H. L. Zhang, D. G. Bucknall and A. D. Briggs, $A d v$. Funct. Mater., 2004, 14, 1081.

14 P. J. Yoo and H. H. Lee, Macromolecules, 2005, 38, 2820.

15 S. K. Basu, A. M. Bergstreser, L. F. Francis, L. E. Scriven and A. V. McCormick, J. Appl. Phys., 2005, 98, 063507.

16 S. K. Basu, L. E. Scriven, L. F. Francis, A. V. McCormick and V. R. Reichert, J. Appl. Polym. Sci., 2005, 98, 116.

17 S. K. Basu, L. E. Scriven, L. F. Francis and A. V. McCormick, Prog. Org. Coat., 2005, 53, 1.

18 R. Huang, C. M. Stafford and B. D. Vogt, MRS Online Proc. LIbr., 2006, 924, 0924-Z04-10.

19 Z. Y. Huang, W. Hong and Z. Suo, J. Mech. Phys. Solids, 2005, 53, 2101.

20 S. Cai, D. Chen, Z. Suo and R. C. Hayward, Soft Matter, 2012, 8, 1301.

21 E. S. Matsuo and T. Tanaka, J. Chem. Phys., 1988, 89, 1695.

22 T. Hwa and M. Kardar, Phys. Rev. Lett., 1988, 61, 106.

23 T. Tanaka, S. T. Sun, Y. Hirokawa, S. Katayama, J. Kucera, Y. Hirose and T. Amiya, Nature, 1987, 325, 796.

24 T. Tanaka and D. J. Fillmore, J. Chem. Phys., 1979, 70, 1214.

25 K. Sekimoto and K. Kawazaki, Phys. A, 1989, 154, 384.

26 A. Onuki, Phys. Rev. A: At., Mol., Opt. Phys., 1989, 39, 5932.

27 M. Doi and A. Onuki, J. Phys. II, 1992, 2, 1631.

28 T. Yamaue and M. Doi, J. Chem. Phys., 2005, 122, 084703.

29 Y. Li, C. Li and Z. Hu, J. Chem. Phys., 1994, 100, 4637.

30 M. Guvendiren, J. A. Burdick and S. Yang, Soft Matter, 2010, 6, 5795 .

31 J. Yoon, J. Kim and R. C. Hayward, Soft Matter, 2010, 6, 5807.

32 E. S. Matsuo and T. Tanaka, Nature, 1992, 358, 482.

33 L. Pauchard and C. Allain, Europhys. Lett., 2003, 62, 897.

34 R. Rizzieri, L. Mahadevan, A. Vaziri and A. Donald, Langmuir, 2006, 22, 3622.

35 H. H. Wang, T. W. Shyr and M. S. Hu, J. Appl. Polym. Sci., 1999, 73, 2219.

36 P. G. De Gennes, Eur. Phys. J. E: Soft Matter Biol. Phys., 2002, 7, 31.

37 F. Doumenc, B. Guerrier and C. Allain, Polymer, 2005, 46, 3708.

38 P. J. Flory and J. J. Rehner, J. Chem. Phys., 1943, 11, 521.

39 L. Leibler and K. Sekimoto, Macromolecules, 1993, 26, 6937.

40 F. N. Kelley and F. Bueche, J. Polym. Sci., 1961, 50, 549.

41 T. S. Chow, Macromolecules, 1980, 13, 362.

42 DSC measurements shows an increase in $T_{\mathrm{g}}$ from $77^{\circ} \mathrm{C}$ to $129^{\circ} \mathrm{C}$ between non-crosslinked PVA and a crosslinking ratio of $10 \mathrm{~mol} \%$.

43 S. Kopp-Marsaudon, Ph. Leclère, F. Dubourg, R. Lazzaroni and J. P. Aimé, Langmuir, 2000, 16, 8432.

44 A. Onuki, J. Phys. Soc. Jpn., 1988, 57, 699. 